\title{
Morphodynamische Aktivitäts- und Stabilitätszeiten statt Pluvial- und Interpluvialzeiten
}

\author{
Von Heinrich Rohdenburg, Gießen
}

Mit 2 Abbildungen

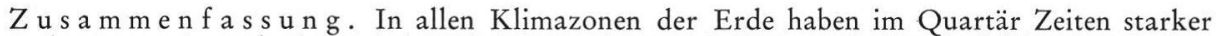
Hangabtragung (morphodynamische Aktivitätszeiten) mit Zeiten der Bodenbildung (morphodynamische Stabilitätszeiten) alterniert. Das Holozän ist eine Zeit morphodynamischer Stabilität infolge zu dichter Vegetation in den niederschlagsreichen Gebieten und zu geringer bzw. zu gleichmäßig verteilter Niederschläge in den vegetationsarmen Gebieten. Die ökologischen Bedingungen für morphodynamische Aktivität können deshalb nicht auf aktualistischem Wege ermittelt werden. Es wird erschlossen, daß die vorzeitlichen Aktivitätsbedingungen in den Subtropen und Tropen nur durch eine stärker akzentuierte Niederschlagsverteilung verursacht worden sein können, denn nur dadurch kann sowohl die Leistungsfähigkeit der Vegetation verringert als auch der Oberflächenabfluß wesentlich erhöht worden sein. Eine Veränderung der absoluten Niederschlagsmenge ist dagegen erst in zweiter Linie wichtig; wahrscheinlich waren die Niederschläge in den von dichter Vegetation bedeckten Gebieten niedriger und in den ariden Gebieten höher als heute. Der klassische Pluvialbegriff muß aufgegeben werden, da in ihm in den semiariden und ariden Gebieten alle vermeintlichen Anzeichen für vorzeitlich gesteigerte (oft aber nur andersartige) Wasserwirkung zusammengefaßt worden sind, ohne zwischen einer Verbesserung des Bodenwasserhaushaltes einerseits und einer Steigerung der Hangabtragung durch wesentlich verstärkten Oberflächenabfluß andererseits zu unterscheiden. Diese beiden Möglichkeiten der Veränderung des Wasserhaushaltes führen jedoch $\mathrm{zu}$ den grundsätzlich voneinander verschiedenen ökologischen Zuständen der morphodynamischen Stabilität und Aktivität, die nach bodenstratigraphischen Untersuchungen sowohl in den Subtropen als auch in den Tropen nie gleichzeitig verwirklicht waren.
\end{abstract}

$\mathrm{Su} \mathrm{m}$ m a $\mathrm{y}$. In the course of the quaternary, all climatic zones of the world were marked by an alternation of extensive slope erosion (periods of morphodynamic activity) and soil formation (periods of morphodynamic stability). The holocene is a period of morphodynamic stability owing to overdense vegetation in areas of abundant rainfall and insufficient or too evenly distributed rainfall in areas with reduced vegetation. Thus, the ecological conditions relevant for morphodynamic activitiy are not discoverable by using the actualistic approach. The pleistocene conditions of activity prevailing in the tropical and subtropical areas must necessarily be due to less even rainfall distribution since only this is sufficient to explain both a less luxuriant vegetation and a substantially increased ratio of surface runoff. However, the question as to wether this involved a change in the absolute rainfall amount is of secondary importance only; it is likely that rainfall was lower in areas covered with dense vegetation and greater in areas of great aridity than now is the case. The time-honoured pluvial concept must be abandoned, since in the case of the semiarid and arid areas it lumps together all of the alleged indications of a former increase (often, however, only of a different type) in the effects of precipitation without ever distinguishing between an improved soil water balance on the one hand and an increase in slope erosion produced by a substantially higher ratio of surface runoff on the other. It is these two possibilities leading to changes in the water balance that result in the essentially different ecological states of morphodynamic stability and activity, which soil stratigraphy has revealed never to be coeval in either the tropical or subtropical areas.

R é s u mé. Pendant le Quaternaire toutes les zones climatiques ont été marquées par une alternance de périodes de forte érosion de pentes (périodes morphodynamiques actives) et de formation de sol (période morphodynamiques stables). A cause de la végétation trop dense dans les régions à forte précipitation, et d'un manque de pluie, voire un régime à pluies trop régulier dans les régions pauvres en végétation, l'holocène représente une période à stabilité morphodynamique. Ainsi, les conditions écologiques de l'activité morphodynamique ne peuvent être découvertes en étudiant les processus actuels. 
C'est pourquoi que l'on peut supposer qu'un régime de pluie plus accentué encore, ait engendré les processus d'érosion préholocènes dans les régions tropicales et subtropicales. Car en effet, c'est le seul moyen pour diminuer l'influence de la végétation d'une part et d'accélerer le ruissellement d'autre part. Par contre, un changement de la quantité absolue des précipitations est d'une importance secondaire; probablement, la quantité des précipitations dans les régions à couverture végétale dense, était moindre et dans les régions arides, plus haute qu'aujourd'hui. Il en resulte que le terme "Pluvial", devenu classique, doit être abandonné, puisqu'il renferme soi-disant tous les indices d'une activité fluviale plus élevée (bien que souvent simplement changée), dans les régions arides et semi-arides; d'une part il ne fait aucune différence entre une éventuelle amélioration intervenue dans le budget de l'eau du sol, ni d'autre part, d'une augmentation de l'érosion des pentes dûe à un ruissellement plus intense. Mais ces deux possibilités de changement du budget de l'eau mènent aux deux états écologiques, c. à. d. de stabilité et d'activité morphodynamiques, fondamentalement différents l'un de l'autre; ils ne se réalisent, d'après les recherches pédo-stratigrafiques, jamais en même temps ni dans les subtropiques ni dans les tropiques.

\section{Aktualistische Paläoklima-Konzeptionen}

In der Frühphase der Geomorphologie galt die Überzeugung, daß Abtragung zwar nicht klimaunabhängig, aber doch in allen Klimazonen ständig vor sich gehe und im wesentlichen durch Tektonik beeinflußt sei. Heute wird in viel stärkerem Maße eine klimatische Abhängigkeit der Abtragung anerkannt. Als eines der wichtigsten Ergebnisse dieser klimagenetischen Geomorphologie ist anzusehen, daß es Zeiten $\mathrm{klimabedingter} \mathrm{Abtragungsruhe} \mathrm{gibt,} \mathrm{und} \mathrm{zwar} \mathrm{selbst} \mathrm{in} \mathrm{Hebungsgebieten}$ mit starkem Relief. Die Vorstellung eines klimabedingten Alternierens von Zeiten der Abtragungsruhe mit solchen starker Abtragung ist in Mitteleuropa besonders einleuchtend, da einerseits der natürliche Laubmischwald der pleistozänen Warmzeiten und des Holozäns - natürlich nur bei anthropogen ungestörter Vegetation - einen guten Bodenschutz gewährleistet und andererseits in den pleistozänen Kaltzeiten durch ein Periglazialklima mit stark bodenwirksamer Frostdynamik ein anderes Abtragungsregime herrschte, dessen Abtragungswirksamkeit schon durch die größtenteils lückige Vegetationsdecke manifestiert wird.

Anders liegen die Verhältnisse außerhalb der pleistozän-kaltzeitlichen Periglazialgebiete, also in den Subtropen und Tropen. Für diese Gebiete wurde noch lange und wird z. T. noch heute die alte Vorstellung der ständig weitergehenden Formung aufrechterhalten. Infolgedessen wird das Relief in den Tieflagen der Subtropen und Tropen häufig noch heute nur mit den klimatischen Verhältnissen der Gegenwart in Beziehung gebracht, wenn die Formendifferenzierung erklärt werden soll. Eine klimagenetische Fragestellung erscheint nach dieser Auffassung nicht ergiebig; deshalb kann der diesen Zonen fehlende, z. T. sogar deutlicher als in Mitteleuropa ausgeprägte Stockwerkbau des Reliefs dort nur tektogenetisch erklärt werden. In unserem Zusammenhang ist also wichtig, daß im Gegensatz zu Mitteleuropa in diesen Zonen vielfach weder für die Gegenwart noch für frühere Zeiten mit zeitweiliger Abtragungsruhe gerechnet wird. Eine Ausnahme machen allerdings die Vorländer von Gebirgen — und natürlich diese selbst —, die im Pleistozän große Areale mit Periglazialmilieu aufgewiesen haben und auf die wegen dieser Nachbarschaftswirkung schon seit längerem klimagenetische Fragestellungen angewandt worden sind. Insbesondere wurde die Pedimentbildung in den subtropischen Trockenräumen lange nur unter diesem Gesichtspunkt gesehen.

Heute ist jedoch allen geomorphologisch orientierten Bodenkundlern sowie allen bodenkundlich informierten Geomorphologen deutlich, daß es überall auf der Erde, also auch in den wechsel- und immerfeuchten Tropen (vgl. u. a. Fölster 1965, 1969, RohDEnburG 1969), ebenso ausgeprägte klimabedingte Formungswechsel gegeben hat wie in Mitteleuropa: Überall alternierten Zeiten starker Hangformung mit solchen der Abtragungsruhe. Im Mittelmeergebiet sind schon lange vorzeit- 
liche Hangschuttdecken auch in den Tieflagen bekannt (u. a. Mensching 1955, Wiche 1961, Butzer 1964a u. b, Rohdenburg \& Sabelberg 1969a u. b). Außerdem treten Fußflächen, bzw. Fußflächentreppen auch am Fuß niedriger Gebirge auf, deren Bildung nicht auf die Nachbarschaftswirkung einer periglazialen Höhenstufe zurückgeführt werden kann (Wiche 1959, 1961, Mensching 1964a). Diese Hangschuttdecken und Tieflagenpedimente werden allgemein durch die Annahme eines feuchteren ("pluvialen“) Klimas erklärt, in dem stärkere Oberflächenabspülung wirksam gewesen sein soll. Als Anzeichen eines vorzeitlich feuchteren Klimas werden auch frühere Seehochstände angesehen, die offensichtlich zeitgleich mit der Vergletscherung im Gebirge und der Hangschutt- und Pedimentbildung in den Tieflagen (z. B. Coque 1962, Mensching 1964b) sind.

In den Tropen werden ebenfalls verbreitet Anzeichen ehemals weit stärkerer Abtragung gefunden, so z. B. Pedimenttreppen, Schutthorizonte und Steinsohlen sowie vorzeitlich viel breitere Flußbetten als in der Gegenwart (Rohdenburg 1969, 1970). Sowohl in Südamerika (Bigarella 1966, Mabesoone 1966) als auch in Afrika (u.a. BrücKner 1955, Fölster 1964, 1969, de Ploey 1965, Rohdenburg 1969, 1970, Tricart 1965, Vincent 1966, Vogt 1966) werden diese Phasen starker Abtragung als Zeiten eines gegenüber heute wesentlich $\mathrm{trock}$ en e re n Klimas mit weniger dichter Vegetation gedeutet ${ }^{1}$ ), da die gegenwärtige Vegetation nicht mit stark wirksamer, mehr als nur Feinmaterial verspülender Abtragung vereinbar ist. Betont werden muß, daß es in dieser Hinsicht keine wesentlichen Unterschiede zwischen den Gebieten mit Regenwald und denjenigen mit Trockenwald (Savanne) gibt, wenigstens soweit dies Westafrika betrifft.

Dieser Überblick zeigt, daß die unabhängig voneinander entwickelten paläoklimatischen Konzeptionen der Subtropen einerseits und der Tropen andererseits einander völlig entgegengesetzt argumentieren. Dabei fällt auf, daß in den die Subtropen betreffenden Arbeiten kaum Vorstellungen über die Vegetation der Zeiten starker Hangabtragung entwickelt worden sind. In einer der wenigen Stellungnahmen dazu äußert WICHE (1961, S. 148), daß der pluvialzeitliche Wald keineswegs dichter als derjenige der Gegenwart gewesen sein könne, vor allem aber sei er nicht mit zusammenhängendem Bodenbewuchs ausgestattet gewesen. Auch ButZer (1961) rechnet trotz der angenommenen Niederschlagserhöhung nicht mit einer dichteren Vegetation als heute, wobei er die heutigen Vegetationstypen der Garriguen und der "light oak woodlands" mit „incomplete soil protection“ für die natürliche Vegetation hält. Wenn man jedoch berücksichtigt, daß diese Vegetationstypen durch starke anthropo-zoogene Beeinflussung aus Wäldern mit sehr viel besserem Bodenschutz hervorgegangen sind, dann hätten WICHE und BUTZER für die „pluvialzeitliche" Vegetation eine Auflichtung gegenüber der heutigen natürlichen Vegetation fordern müssen. Diese kann dann aber nicht mehr durch die Annahme einer „pluvialzeitlichen“ Niederschlagsvermehrung erklärt werden.

Butzer hat allerdings ein vermeintliches Indiz für relativ humide Verhältnisse während der Ablagerung der Hangsedimente aufgeführt, nämlich ihre häufige Wechsellagerung mit Kalkkrustenlamellen, die er als sinterähnliche Ablagerung aus einer Zeit mit stärkerer Quellaktivität entstanden denkt. Aber auch dieses Argument für eine Niederschlagsvermehrung ist nicht stichhaltig, denn die Kalkkrustenlamellen sind nicht während der Sedimentation gebildet worden, sondern sie sind in diesen Sedimenten nachträglich als Teile eines vielgliedrigen $\mathrm{Ca}$-Horizontes von einer höheren Oberfläche aus und in einer Zeit

1) Von bodenkundlicher Seite sind Steinsohlen und Schutthorizonte zunächst als subterran durch Termitentätigkeit und Bodenfließen verursachte Erscheinungen angesehen worden (NYE 1954, 1955). Diese Ansicht ist heute hinfällig, da die subaerische Entstehung der Grobmaterialkörper ausreichend belegt ist und außerdem deutlich wird, daß auf flachen Böschungen kein tiefgründiges Bodenfließen erfolgt. 
mit kräftiger Bodenbildung entstanden (Rohdenburg \& Sabelberg 1969a, b²). Auch die höheren Seespiegel sind keine eindeutigen Indikatoren höherer Niederschläge, denn wie Haude (1969) am Beispiel des Toten Meeres nachweisen konnte, müßte der Seespiegel in kühleren Zeitabschnitten auch dann erheblich ansteigen, wenn die Niederschlagsmenge gleichbliebe, da die Verdunstung infolge der Temperaturerniedrigung stark verringert war (vgl. auch Frenzel 1967). Wenn man jedoch eine höh e re Abflu ß quote annimmt - etwa durch einen größeren Starkregenanteil am Niederschlag - , dann hätten die Seespiegel sogar bei geringerer Niederschlagsmenge höher stehen können (s. u.).

Für „pluvialzeitliche“ Niederschlagserhöhung im Mittelmeergebiet schien jedoch noch ein indirektes Argument zu sprechen: Trockenzeiten waren nach allgemeiner Auffassung schon als Entstehungszeiten der verbreiteten Kalkkrusten „vergeben“. Nun beobachtet man generell ein Alternieren von Schutthorizonten und Kalkkrusten; in der Überzeugung, es habe ein Alternieren von Trocken- und Feuchtzeiten gegeben, stellte man die Kalkkrusten in die Trockenzeiten, die Hangschuttdecken folglich in die Feuchtzeiten. Heute ist jedoch klar, daß die Kalkkrusten keine Oberflächenbildungen sind, sondern Ca-Horizonte kräftig entwickelter Böden und klimatisch somit als Fe u cht$\mathrm{z}$ e itbild ungen angesprochen werden müssen (RohDenburg \& SAbelberg 1969a, b). Auch von diesem Aspekt aus wäre also der Weg frei, die morphodynamisch aktiven Zeiten als relative Trockenzeiten anzusehen.

Haben sich einerseits also alle angeblichen Feuchtzeitindikatoren als nicht aussagekräftig erwiesen, so gibt es andererseits heute positive Hinweise für kaltzeitliche Trockenzeiten, nämlich erstens Lößvorkommen im Mittelmeergebiet (VIRGILI \& ZAMARreño 1957, Riba 1957, Alia Medina \& Riba 1957, Rohdenburg \& Sabelberg 1969a, b, BrunNaCKer 1969, BrunNaCKer \& LožEK 1969) und zweitens das überraschende Ergebnis der in den letzten Jahren durchgeführten Pollenanalysen aus Mittelitalien (BonatTI 1962), Südspanien (MenÉndez AmOr \& Florschütz 1964) und Griechenland (van DER Hammen, Wijmstra \& van Der Molen 1965), daß es im Mittelmeergebiet in der letzten Kaltzeit recht offene Vegetationstypen und zeitweilig sogar völlig waldfreie Abschnitte gegeben hat, wie BEUG (1968) eindrucksvoll zusammenstellte.

Demnach besteht also in neuerer Sicht kein grundsätzlicher Unterschied mehr zwischen Tropen und Subtropen hinsichtlich der paläoklimatischen Vorstellungen der Zeiten mit starker Hangabtragung. In beiden Zonen müssen nämlich Klimaverhältnisse geherrscht haben, die keine geschlossene Vegetationsdecke, sondern relativ offene Vegetationstypen mit geringem Bodenschutz bedingten. Gegenüber der älteren Auffassung, die der Vege-

2) Sickerwasser kann häufig nicht undifferenziert in den Boden eindringen, sondern es findet eine Differenzierung in eine Vielzahl von dünnen Wasserhorizonten statt. Wenn nach einem Niederschlag die Bodenluft in Ermangelung von kontinuierlichen groben Poren nicht rasch genug nach oben entweichen kann, sondern von der Sickerwasserfront eingeschlossen wird, kommt es schließlich zu einem Sickerwasserstau auf komprimitierten "Luftkissen“, d. h. zur Bildung von "Wasserbändern“ (im zweidimensionalen Profilbild) (Meyer \& Moshrefi 1969) mit hoher bis vollständiger Porenfüllung durch Wasser, die im großen oberflächenparallel angeordnet sind. Infolge der wachsenden Sickerwasserauflast kommt es lokal zu einem Durchbrechen der Luftkissen - nicht zu deren Auflösung -, jedoch in etwas größerer Tiefe zur Wiederholung der Wasserbänder-Luftkissenbildung. Die Sickerwasserbewegung ist nur im großen vertikal gerichtet, im einzelnen herrscht in den Wasserbändern laterale Bewegung - hangabwärts oder zu Durchbruchstellen - vor. Entsprechend dieser Sickerwasserdifferenzierung weisen auch Akkumulationshorizonte häufig einen lagigen Aufbau auf, wie z. B. Eisen- und Humusbänder in Podsolen und Tonbänder in Parabraunerden ("Tüxen-Bänder“). Die Kalklamellenserien der Kalkkrusten stellen eine vollständig analoge Differenzierung von Ca-Horizonten dar. Mit der Luftkissen-Wasserbänder-Theorie von Meyer \& Moshrefi (1969) läßt sich also sowohl die Vielzahl der gleichzeitig übereinander gebildeten Lamellen als auch die laterale Wasserbewegung erklären, die für die Bildung der Kalklamellen stets betont worden ist und für die bislang nur eine oberflächliche Entstehung in Frage zu kommen schien. 
tation keine wesentliche Beeinflussung der Abtragung zugestand, geht also heute die Tendenz eher dahin, eine sehr enge Abhängigkeit zwischen Vegetation und Formung anzunehmen und Klimawechsel vor allem nach dem Ausmaß ihrer Vegetationsveränderung $\mathrm{zu}$ beurteilen. Aber auch diese Ansicht kann nicht voll zutreffen, wie besonders gut anhand der Verhältnisse in den extremen Trockengebieten gezeigt werden kann.

\section{Hinweise auf nicht aktualistisch deutbare Paläoklimate}

Nach den vorangegangenen Ausführungen könnte man nämlich erwarten, daß die geomorphologische Aktivität mit der Zunahme der Aridität und der entsprechenden Abnahme der Vegetationsdichte zunimmt, also z. B. sowohl von Norden wie von Süden zur Sahara hin. Das ist zwar in gewissem Umfange der Fall, da in dieser Richtung die Breite der Talböden zunimmt und somit, z. B. am Fuß von Schichtstufen, ein relativ großer Teil des Reliefs dem Bereich starker rezenter Formung angehört. Aber gerade in diesen Gebieten wird auch heute noch wie selbstverständlich mit der Pluvialhypothese gearbeitet (vgl. Mensching 1964b) und offensichtlich nicht zu unrecht. Am Nord- und Südsaum der Sahara ebenso wie in der Zentralsahara (vgl. Rognon 1967, sowie Butzer \& Hansen 1968) zeigt sich nämlich, daß hier heute keine allgemeine Pedimentation stattfindet, wie sie vorzeitlich sowohl in diesen wie auch in den feuchteren Gebieten zeitweilig herrschte. Das demonstrieren auch sehr gut die Beobachtungen Hövermanns (1967) und Hagedorns (1967) aus dem Tibestigebiet, wo heute im Gegensatz zu früheren Verhältnissen Inselberge und Flächen nicht in einem Formungszusammenhang stehen, sondern häufig durch Randfurchen voneinander getrennt werden.

In diesem Zusammenhang sind die Messungen der Schwebstofführung in Flüssen sehr aufschlußreich, die Herrmann (1969) im Gebiet der klimatisch außerordentlich stark differenzierten Sierra Nevada de Santa Marta (Kolumbien) durchgeführt hat. Wie zu erwarten war, steigt die Schwebstofführung mit abnehmender Niederschlagsmenge infolge des abnehmenden Bodenschutzes stark an, sinkt jedoch nach einem Maximum in der Zone des trockenkahlen Tieflandswaldes wieder $a b$, da im Bereich der offeneren Vegetations-

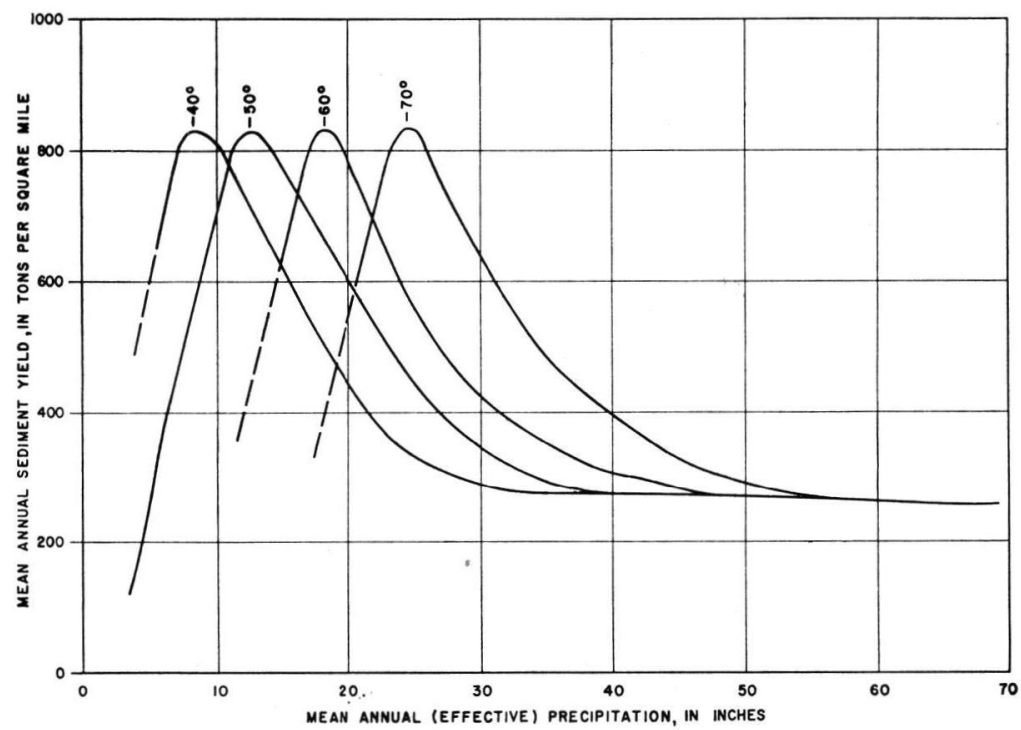

Abb. 1. Rezente Abtragung in Abhängigkeit von Niederschlagsmenge und Temperatur in den USA (aus Sснимм 1965: fig. 2). 
typen die Starkregenwirkung (Produkt aus Intensität und Dauer) geringer ist als in den etwas niederschlagsreicheren Gebieten. Aber selbst im Maximumgebiet der heutigen Abtragung ist die Abtragungswirkung sehr gering (heute fast nur Schwebstoffabtragung) im Vergleich zu einer vorzeitlichen Phase starker morphodynamischer Aktivität mit beträchtlicher Grobmaterialabfuhr sowohl an Hängen als auch in den Flüssen (BARTELS 1970). Eine Zusammenfassung der Abtragungsmessungen des Geological Survey der USA durch LANGbein \& Schumm (1958) weist ebenfalls ein ausgesprochenes Maximum auf, das nach Schumm (1965) dort liegt, wo sich keine geschlossene Grasdecke mehr ausbilden kann (vgl. Abb. 1). Sснuмm (1965) benutzt nun dieses Diagramm, um aufgrund als bekannt vorausgesetzter Werte der pleistozänen Temperaturerniedrigung und der Niederschlagsveränderung detaillierte Angaben über die hydrologischen Bedingungen während der letzten Kaltzeit in unterschiedlichen Gebieten der USA machen zu können. Dieser rein aktualistische paläohydrologische Versuch muß aber a priori zum Scheitern verurteilt sein, da es im Pleistozän Abtragungsbedingungen gab, die heute in den USA nicht gegeben sind (nicht nur Schweb-, sondern auch starke Grobmaterialabfuhr sowohl an Hängen als auch in den Flüssen) und deshalb auch in dem hier wiedergegebenen Diagramm nicht enthalten sein können.

Es zeigt sich also überall, daß in den feuchteren Gebieten heute die Vegetation zu dicht ist für starke Abtragung, daß in den Trockengebieten mit ihrem weitaus geringeren Bodenschutz jedoch nicht genügend morphodynamisch wirksame Niederschläge fallen. Angesichts dieser Problemlage, daß nämlich eine einfache Verschiebung der heutigen Klimazonen auf keinen Fall ausreicht, die Phasen starker vorzeitlicher Morphodynamik zu erklären, müssen dafür zwangsläufig Klimaverhältnisse angenommen werden, die es zwischen Mitteleuropa und dem Golf von Guinea, zwischen den Feuchtwald- und den Trockengebieten im nördlichen Südamerika, sowie zwischen den humiden und den ariden Gebieten der USA in der Gegenwart nicht gibt. ${ }^{3}$ ) $\mathrm{Da}$ es außerdem den Anschein hat, als habe starke geomorphologische Aktivität in sehr großen Räumen gleichzeitig geherrscht, wird man nicht nur mit einem einheitlichen Klimatyp von hoher Abtragungsgunst rechnen dürfen, sondern diesem sogar eine erhebliche Variationsbreite zugestehen müssen.

\section{Stabilitäts- und Aktivitätszeiten}

Wenn man diese Voraussetzungen berücksichtigt, dann kann die Veränderung gegenüber der vorhergegangenen Phase mit verbreiteter Abtragungsruhe nur aus einer Faktorenveränderung resultieren, die in den vegetationsstabilisierten Gebieten $\mathrm{zugleich}$ den Oberflächenabfluß vermehrt und die Vegetation schädigt. Beide Bedingungen werden z. B. durch eine Temperaturerniedrigung erfüllt, wenn diese so stark ist, daß Periglazialmilieu erreicht wird. Für die Gebiete außerhalb der kaltzeitlichen Periglazialzone trifft dieser leicht verstehbare Fall nicht zu, dort genügen einfache Faktorenveränderungen (Temperaturerniedrigung, Niederschlagserniedrigung, Niederschlagserhöhung) nicht b e i d e n genannten Bedingungen. Vielmehr kann außerhalb des vorzeitlichen Periglazialbereichs nur eine weniger gleichmäßige Niederschlags verteilung sowohl die Leistungsfähigkeit und damit die bodenschützende Wirkung der Vegetation beeinträchtigen als auch den Oberflächenabfluß fördern.4) Das soll anhand der

3) Interessant ist, daß auch BüDEL (1963) trotz seiner aktualistischen Vorstellung von der vorzeitlichen Morphodynamik sich zu einer nicht aktualistisch deutbaren Paläoklima-Konzeption gezwungen sieht.

4) In diesem Zusammenhang ist wichtig, daß FloHN (1970) für die pleistozänen Kaltzeiten folgert, daß der konvektive Charakter der Niederschläge über den tropischen und subtropischen Kontinenten noch zugenommen hat, was in einer Akzentuierung der Niederschlagsverteilung resultieren dürfte. 
ökologischen Verhältnisse bei zunehmender Akzentuierung der Niederschlagsverteilung näher erläutert werden, die sich allerdings nur auf die gegenwärtig vegetationsstabilisierten Gebiete (über $100-150 \mathrm{~mm}$ ) beziehen soll: Bei schwachen Einzelregen und einer annähernd gleichmäßigen Niederschlagsverteilung über das Jahr, wie sie heute für humide Gebiete charakteristisch ist, wird fast der gesamte auf die Bodenoberfläche gelangende Teil des Niederschlags (Niederschlag minus Interception) in den Boden eindringen und dort entweder durch die Vegetation aufgenommen werden oder ins Grundwasser gelangen. Die Vegetation wird also bei dieser Niederschlagsverteilung optimal mit Wasser versorgt, während nur ein Bruchteil des Niederschlags als Oberflächenwasser abfließt (z. B. in den Mittelbreiten während der Schneeschmelze), der dichten Vegetation wegen aber kaum Abtragungswirkungen hervorrufen kann. Diese Niederschlagsverteilung ist zugleich optimal für starke Mineralverwitterung und weitere chemische Umsetzungen in Oberflächennähe (Bodenbildung) und in größerer Tiefe (Saprolithisierung).

Je stärker die Einzelregen und je unregelmäßiger die Niederschlagsverteilung während des Jahres, bzw. je stärker die Niederschlagsmengen von Jahr zu Jahr schwanken, desto ungünstiger ist die Wasserversorgung der Vegetation. Erstens geht ein größerer Teil des Niederschlagswassers für die Vegetation verloren, weil es bei Starkregen nicht sämtlich einsickert, sondern z. T. oberflächlich abfließt. Zweitens wird die Leistungsfähigkeit der Vegetation durch längere Trockenzeiten stark verringert; die Vegetation ist zwar trokkenheitsangepaßt, d. h. sie kann Trockenzeiten überstehen, aber ihre Assimilation ist stark eingeschränkt und außerdem kann bei xeromorphen Pflanzen ein geringerer Teil der Assimilate für die Vergrößerung der Blattmasse und damit der Assimilation eingesetzt werden als bei mesophytischen. Schon kleine Veränderungen im Sinne eines Ungleichmäßigerwerdens der Niederschläge können relativ große Wirkungen hervorrufen, denn die Schwächung der Leistungsfähigkeit der Vegetation bedeutet schlechteren Bodenschutz gegenüber dem Niederschlag, mehr Oberflächen- aber weniger Sickerwasser und folglich — im Sinne eines Selbstverstärkungsvorganges - eine schlechtere Wasserversorgung der Vegetation. Zugleich steigt aber mit dem Anwachsen der Oberflächenabspülung die Abtragungswirkung. Stärkere Abtragung kann jedoch wiederum die Kampfkraft der Vegetation verringern, erstens durch direkte Wirkungen wie etwa teilweise Freispülung des Wurzelsystems oder sogar Ausspülen von kleineren, aber für den Bodenschutz sehr wichtigen Pflanzenindividuen und zweitens durch eine Beeinträchtigung der Nährstoffversorgung, denn bei vielen Vegetationstypen ist ein sehr großer Teil der leicht verfügbaren Nährstoffvorräte des OKkosystems in der organischen Auflage und im oft geringmächtigen $\mathrm{A}_{\mathrm{h}}$-Hor. enthalten, also an die unmittelbare Oberflächennähe gebunden, so daß schon mäßige Abtragung $\mathrm{zu}$ relativ hohen Nährstoffverlusten führen kann. Die dadurch eintretende Schwächung der Kampfkraft der Vegetation kann somit - in einem zweiten Selbstverstärkungsprozeß - Oberflächenabfluß, Abtragung, Vegetationsschädigung usw. verstärken.

Bei einem Klimawechsel von gleichmäßiger zu immer ungleichmäßig werdender Niederschlagsverteilung wird durch die beiden dargestellten Selbstverstärkungsprozesse innerhalb einer relativ geringen Spanne ein „Umkippen“ des ökologischen Systems erfolgen von einem ersten Zustand mit sehr hohem Sickerwasseranteil, gutem Bodenschutz durch die Vegetation und geringer Abtragung zu einem zweiten Zustand mit lückiger Vegetation, geringem Sickerwasseranteil und starker Abtragung. Der erste Zustand soll im folgenden als Zustand morphodynamischer $\mathrm{Stabilität} \mathrm{bezeichnet} \mathrm{werden,} \mathrm{während} \mathrm{für} \mathrm{den}$ zweiten Grundzustand morphodynamische Aktivität kennzeichnend ist. Stabilitätszeiten sind außer durch dichte Vegetation und geringe Abtragung dadurch gekennzeichnet, daß in ihnen B oden bild ung erfolgt, während die Möglichkeiten dazu in den Aktivitätszeiten nur in sehr viel geringerem Maße oder gar nicht gegeben sind. Da Vegetation in ihnen jedoch nicht völlig fehlt, werden auch pedogenetische Prozesse ablaufen; diese können sich aufgrund ihrer Langsamkeit jedoch nicht im Sinne einer 
deutlichen Bodenbildung ausprägen, wenn gleichzeitig rasche Materialumlagerung durch die Oberflächenabspülung stattfindet; allerdings ist nicht auszuschließen, daß die nicht an Oberflächennähe gebundene Saprolithisierung (Tiefenverwitterung) auch in den Aktivitätsphasen weitergeht. Von der Bodenoberfläche ausgehende Bodenbildung kann jedoch nur in einem durch Oberflächenstabilität gekennzeichneten Okosystem stattfinden. In Akkumulationsstandorten sind deshalb in Sedimente eingeschaltete gut entwickelte B ö de n die besten Indikatoren für vorzeitliche Stabilitätszeiten.

Einen Widerspruch zu dieser Auffassung könnte man darin erblicken, daß die heutige Vegetation an sehr verschiedene Typen der Niederschlagsverteilung so erfolgreich angepaßt ist, daß außerhalb der Wüsten überall ausreichender Bodenschutz für Bodenentwicklung und morphodynamische Stabilität gewährleistet ist. Diese Anpassung ist jedoch erst durch die Klimaentwicklung "provoziert" worden. Sicher haben alle Abschnitte der Klimazyklen Auswirkungen auf die Floren- und Vegetationsentwicklung gehabt, aber vermutlich erfolgte optimale Anpassung nur für die Abschnitte mit der größten Leistungsfähigkeit der Vegetation, also für diejenigen mit der gebietsspezifisch gleichmäßigsten Niederschlagsverteilung und somit der günstigsten Wasserversorgung. Die geringere bzw. fehlende Anpassung der Vegetation eines bestimmten Gebietes an die Klimazyklusabschnitte mit schlechterer Bodenwasserversorgung konnte durch die mit den Klimaschwankungen verbundenen Verschiebungen der Vegetationszonen nicht voll kompensiert werden. Es werden ja nicht alle Klimafaktoren gleich stark verändert; so weisen u. a. das Strahlungsklima sowie das für die Niederschlagsbildung wichtige lokale Windfeld wegen der nicht veränderten Lagebeziehungen ortsspezifische Parameter auf. Bekanntermaßen ist ja das pleistozäne Periglazialklima Mitteleuropas mit keinem Periglazialklima der Gegenwart völlig gleichzusetzen, wie sich schon darin äußert, daß es heute kein Periglazialgebiet mit starker, nicht auf Flußnähe beschränkter Lößanwehung gibt. Der Schwellenwert des "Umkippens" vom Stabilitätszustand in den Aktivitätszustand wird deshalb von Klimagebiet zu Klimagebiet außerordentlich unterschiedlich sein - und selbst innerhalb dieser Gebiete bestehen Unterschiede in Abhängigkeit von der Bodenbeschaffenheit ${ }^{5}$ ) und dem Relief. In erster Näherung ist zu erwarten, daß die allgemeine Zirkulation der Atmosphäre sich in den Aktivitätszeiten nicht vollständig von derjenigen der Stabilitätszeiten unterschieden hat, so daß die niederschlagsreichsten Gebiete der Stabilitätszeiten auch in den Aktivitätszeiten relativ hohe Niederschläge erhalten haben. Je größer die Niederschlagsdichte, desto dichter kann auch die Vegetation in demjenigen Zeitpunkt noch sein, in dem im Übergangsbereich von Stabilitäts- und Aktivitätszeit merkliche Abtragung (Teilaktivität) einsetzt. Auch in den Abschnitten mit voller Aktivität wird z. B. in den Tropen die Vegetation dichter gewesen sein als in den Trockenräumen sowohl während der Aktivitäts- als auch der Stabilitätszeiten. Einen eindeutigen Beleg für diese Auffassung erblicke ich darin, daß in der Savannenzone Westafrikas, in der die Stabilitätszeiten-

5) In einem kürzlich erschienenen sehr interessanten Aufsatz sehen VoLK \& GEYGER (1970, Zeitschr. f. Geomorph., N. F. 14, 79-95) „Schaumböden als Ursache der Vegetationslosigkeit in ariden Gebieten" an. Ich kann das Verhalten der Bodenluft in trockenen schluffreichen Böden, das ja stets das Eindringen des Sickerwassers beeinflußt (vgl. Fußn. 2), nur als einen von vielen Standortsfaktoren anerkennen, die das Umkippen von Stabilität zu Aktivität mitbestimmen. Auch in den Trockenräumen des Mittelmeergebietes wären die heute vegetationsfreien Standorte sicher vegetationsbedeckt, wenn der Mensch nicht in den Landschaftshaushalt eingegriffen hätte. Infolge dieser Störung sind natürlich Standorte mit geringer Regenverdaulichkeit am stärksten beeinträchtigt worden, so daß sie zuerst volle Aktivität aufweisen. Andererseits werden solche Standorte am Ende einer Aktivitätszeit die Wiederbesiedlung mit Vegetation am längsten behindern, so daß sie in extremen Trockengebieten auch vollständig vegetationsfrei bleiben können (vgl. die Beispiele aus Südmarokko). Es ist VolK \& GEYGER zu danken, daß sie die nicht zu unterschätzende Bedeutung der Bodenluft für den Wasserhaushalt (vgl. Fußn. 2 sowie Rohdenburg \& Merer 1966, Mitt. d. Dtsch. Bodenkundl. Ges. 5, S. 101) betont und einen so einfachen Indikator für Böden mit geringer Regenverdaulichkeit herausgearbeitet haben. 
Vegetation heute anthropo-zoogen (Brand, Beweidung) in ihrer Leistungsfähigkeit gehemmt ist, stellenweise schon beachtliche Formungsaktivität eintreten kann, selbst wenn das Wassersammelgebiet eine noch recht dichte Vegationsdecke aufweist (RoHDENBURG 1969). Diese Feststellung erleichtert das Verständnis von starker Formungsaktivität in den Gebieten mit großer Leistungsfähigkeit der Vegetation wesentlich, denn dann kann das Ausmaß des Klimawechsels zwischen Stabilitäts- und Aktivitätszeit als geringer veranschlagt werden, als wenn man etwa annehmen müßte, auch in den Regenwaldgebieten hätten in den Aktivitätszeiten heutige Wüsten- oder wenigstens Halbwüstenbedingungen geherrscht.

Die Niederschlagsverteilung, deren Bedeutung für die Vegetation in den Gebieten ohne ausgeprägte Temperaturminima seit der Arbeit LAUERs (1952) allgemein anerkannt ist, stellt nach der hier dargestellten Auffassung zugleich den wichtigsten Klimafaktor für die Steuerung des Verhältnisses von Vegetation zu Abtragung dar. Wie für den Vegetationstyp, so ist auch für das Vegetations-Abtragungs-Verhältnis die absolute Niederschlagsmenge erst in zweiter Linie wichtig. So könnte der Übergang von Stabilität zu Aktivität also auch bei gleichbleibender Niederschlagsmenge erfolgen; allerdings ist es weniger wahrscheinlich, daß bei einer Veränderung der Niederschlagsverteilung die Niederschlagsmenge unbeeinflußt bleibt. In Frage kommen deshalb vorwiegend die beiden Möglichkeiten der Aktivitätssteigerung bei a) zunehmender und b) abnehmender Niederschlagsmenge.

Der Fall a ist verwirklicht, wenn die ungleichmäßigere Niederschlagsverteilung darin besteht, daß bei etwa gleichbleibender Anzahl schwach- und mittelintensiver Niederschläge die Zahl der Starkregen zunimmt. So korreliert z. B. Leopold (1951) die verstärkte Gully-Bildung um die letzte Jahrhundertwende in New-Mexico mit einer Periode vermehrter Sommergewitter (vgl. auch LeOpold, LeOpold \& Wendorf, 1963). Dieser Fall ist wahrscheinlich dort von größter Bedeutung, wo die Vegetation auch bei morphodynamischer Stabilität nicht sehr dicht ist und wo deshalb durch die Starkregen die Leistungsfähigkeit der Vegetation nicht oder nur unbedeutend gesteigert werden kann, d. h. in den ausgesprochenen Trockenräumen.

Die andere Möglichkeit einer stärker akzentuierten Niederschlagsverteilung (b) besteht in einer Verkürzung der Regenzeit bzw. einer Häufung von Trockenjahren, die in der Regel mit einer Verminderung der Niederschlagsmenge einhergeht: Fall b. Abtragungsverstärkend ist eine Regenzeitverkürzung jedoch nur, wenn die Niederschlagsmenge weniger stark abnimmt als die Zahl der humiden Monate, d. h. wenn die Intensität der Einzelregen relativ zur Vegetationsdichte steigt. Andernfalls würde nur eine einfache Verschiebung der bestehenden Vegetationszonen ohne morphodynamischen Effekt erfolgen. Der Fall b erweist sich somit als Vegetationszonenverschiebung zur trockeneren Seite in Kombination mit Fall a, so daß es für unsere Zwecke also nicht von primärer Bedeutung ist, ob insgesamt Niederschlagserhöhung oder-verminderung e in trit .

\section{Quartäre Klimazyklen, ihre Auswirkungen und ihre Verbreitung}

In Abb. 2 ist der Versuch unternommen worden, die prinzipiellen Veränderungen der wichtigsten Wasserhaushaltsgrößen im Verlaufe eines Klimazyklus mit zunächst stärker werdender und dann wieder abnehmender Akzentuierung der Niederschlagsverteilung darzustellen. Dabei soll der oben beschriebene Fall b realisiert sein, d. h. geringerer Gesamtniederschlag in der Aktivitätszeit als in der Stabilitätszeit. Während für hydrologische Zwecke im allgemeinen Oberflächenabfluß und Grundwasserabfluß zusammengefaßt werden, erfordert unsere Betrachtungsweise eine Abtrennung des Oberflächenabflusses, während Evapotranspiration (Verdunstung von Boden und Pflanze) und Grundwasserabfluß wegen ihrer etwa gleichsinnigen Veränderung im Verlaufe eines Klimazyklus zu einer 
übergeordneten Einheit zusammengefaßt werden können. Die wichtigste Eigenschaft des hier postulierten Klimaganges in ökologisch-morphodynamischer Betrachtung ist zweifellos die Gegenläufigkeit der Bereiche von Oberflächenabfluß einerseits und Evapotranspiration und Grundwasserabfluß andererseits. Dabei ist für die Zeichnung des Diagrammes vorausgesetzt worden, daß im Übergangsbereich zwischen Stabilität und Aktivität (bzw. umgekehrt) der Oberflächenabfluß (schraffierter Bereich) viel schneller anwächst als die Niederschlagsmenge absinkt, so daß die Abschnitte mit Teilaktivität also relativ kurz sind. Außerdem wurde aus weiter unten $\mathrm{zu}$ besprechenden Gründen eine ausgeprägte Asymmetrie der "Klimawelle“ angenommen. Für den Oberflächenabfluß in den Stabilitätszeiten wurde ein vermittelnder Wert eingetragen zwischen nahezu Null bei dichter Vegetation sowie sehr gleichmäßiger Niederschlagsverteilung und höheren Werten sowohl

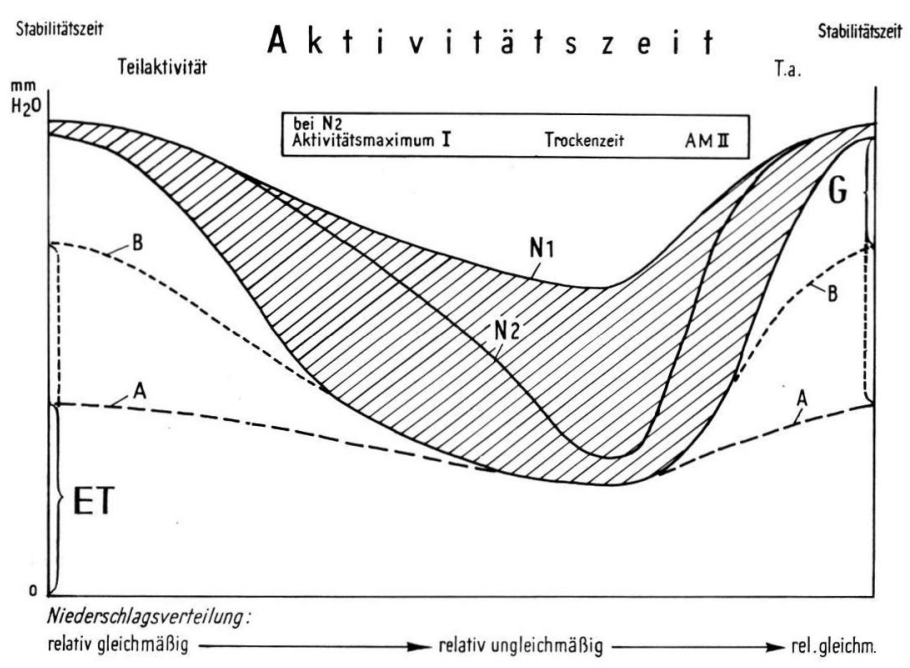

Abb. 2. Oberflächenabfluß (schraffiert), Grundwasserabfluß (G) und Evapotranspiration (ET) im Verlaufe eines Klimazyklus mit zunächst wachsender und später wieder abnehmender Akzentuierung der Niederschlagsverteilung. $\quad \mathrm{N}=$ Niederschlagsmenge; $\mathrm{A}, \mathrm{B}=$ Erläuterung im Text.

in den Trockengebieten als auch in den mit einer dichten Vegetationsdecke versehenen feuchten Tropen aufgrund des in beiden Bereichen hohen Starkregenanteils. Diesen $\mathrm{Zu}-$ stand mit z. T. recht hoher Schwebestoffabfuhr auf recht flachen Böschungen müssen wir entgegen anderen Auffassungen - wie z. B. BüDEL trotz überreichlicher Gegenbeweise (und ohne diese Problematik überhaupt zu diskutieren) noch in neuester Zeit (1970) behauptet - als einen Zustand der S $\mathrm{t}$ a b i l i t ä $\mathrm{t}$ betrachten, denn einmal findet dort heute Bodenbildung statt und zum anderen sind auch in den immer- und wechselfeuchten Tropen die vorzeitlichen Aktivitätszeiten durch Grobmaterialtransport auf allen Böschungen gekennzeichnet (Fölster 1965, 1969, Rohdenburg 1969). Dieser Auffassung entspricht z. B. auch, daß heute in den wechselfeuchten Tropen auf Gesteinen hoher Regenverdaulichkeit (etwa dem grobkörnigen Falsebedded Sandstone der Oberkreide in SE-Nigeria) bei Böschungen unter $5^{\circ}$ keinerlei Oberflächenabfluß stattfindet, während dort in der letzten Aktivitätszeit aufgrund hohen Oberflächenabflusses Pedimentation erfolgte (Herrmann 1968, RohDEnburg 1969, 1970)!

So wie der Oberflächenabfluß der Stabilitätszeiten in Abhängigkeit von Klima, Gestein und Relief außerordentlich stark variiert, so groß sind auch die Unterschiede im Verhältnis zwischen Grundwasserabfluß und Evapotranspiration. Dieser Variationsbreite soll durch die Einzeichnung der beiden Kurven A und B Rechnung getragen werden. Die Kurve 
A trifft zu für Gebiete mit sehr hohen Niederschlagsmengen, in denen trotz hohen Wasserverbrauchs durch die Vegetation der ins Grundwasser gelangende Überschuß an Bodenwasser groß ist. Mit abnehmender Niederschlagsmenge sinkt zwar die Evapotranspiration, aber noch stärker der nicht von der Vegetation verbrauchte Bodenwasserüberschuß, so daß der Grundwasserabfluß schon im dauernd humiden Mittelbreitenklima (etwa Mitteleuropa) recht gering sein kann (etwa Kurve B), in Richtung auf die Trockengebiete jedoch bis auf Null absinkt.

$\mathrm{Da}$ bei den heutigen Bedingungen überwiegender morphodynamischer Stabilität der Oberflächenabfluß überall relativ klein ist, verändert sich auch der Gesamtabfluß gleichsinnig mit der Niederschlagsmenge. Man ist deshalb geneigt, vorzeitlich höhere Seespiegelstände ohne weiteres als Zeiten höherer Niederschläge zu deuten. Diese einfache Beziehung kann jedoch nur für Stabilitäts-, nicht aber uneingeschränkt ebenfalls für Aktivitätsbedingungen mit ihrem wesentlich erhöhten Oberflächenabfluß gelten, wie ebenfalls aus Abb. 2 zu entnehmen ist: In allen normal humiden bis semiariden Gebieten (Grundwasserabfluß gemäß Kurve B oder niedriger) ist zu erwarten, daß beim Übergang zur Aktivitätszeit die Abnahme des Grundwasserabflusses durch die Zunahme des Oberflächenabflusses überkompensiert wird, so daß die Folge ein erhöhter Gesamtabfluß ist. Seespiegelhochstände, die in diesen Gebieten mit einer Aktivitätszeit verknüpft sind, dürften also nicht auf erhöhte Grundwasserspende infolge höherer Niederschläge, sondern auf höheren Oberflächenabfluß infolge stärker akzentuierter Niederschlagsverteilung bei vermutlich sogar verringerten Gesamtniederschlägen zurückzuführen sein. Zwei Klimabereiche fallen jedoch nicht in den Geltungsbereich dieser wichtigen Beziehung:

1. In Gebieten mit stabilitätszeitlich sehr hohem Grundwasserabfluß (etwa Kurve A) aufgrund sehr hoher Niederschläge dürfte im Übergang zu Aktivitätsbedingungen die Abnahme des Grundwasserabflusses nicht völlig von der Zunahme des Oberflächenabflusses kompensiert werden. Dieser Fall ist jedoch von geringer Bedeutung für die Frage vorzeitlicher Seehochstände, da die stabilitätszeitlich niederschlagsreichen Gebiete bezeichnenderweise arm an abflußlosen Becken sind.

2. Extrem aride Gebiete weisen trotz Vegetationsmangels wegen zu geringer Niederschläge nur geringe morphodynamische Aktivität auf (s. o.). Sowohl Zeiten vorzeitlicher Bodenbildung als auch Zeiten vorzeitlich starker morphodynamischer Aktivität, wie sie in manchen dieser Gebiete festgestellt werden (z. B. in Nubien durch Butzer \& HANSEN 1968), können dort nur durch vorzeitlich höhere Niederschläge bedingt sein, die auch einen höheren Abfluß bewirkt haben werden. Dabei ist jedoch anzunehmen, daß der Gesamtabfluß in den Aktivitätszeiten größer war als in den Bodenbildungszeiten, es sei denn, diese wären durch sehr große Humidität gekennzeichnet gewesen. Jeder Versuch einer Analyse höherer Seespiegelstände in ariden Gebieten erfordert also zunächst eine Untersuchung darüber, ob sie entweder in eine Zeit erhöhter morphodynamischer Aktivität oder in eine Zeit mit Bodenbildung fallen.

Die bisherigen Betrachtungen sind davon ausgegangen, daß das Maximum der Niederschlagsverminderung mit dem Maximum der morphodynamischen Aktivität zusammenfällt. Diese Annahme mag für bestimmte Gebiete bzw. für bestimmte Aktivitätszeiten zutreffen (s. u.), so gilt sie nach vielen Geländebeobachtungen jedoch sicher nicht überall bzw. für alle Aktivitätszeiten. Bei sehr ausgeprägter Niederschlagsverminderung mit wachsender Akzentuierung der Niederschlagsverteilung, wie sie in Abb. 2 z. B. durch die Kurve N 2 dargestellt ist, steigt zwar der Oberflächenabfluß zunächst rasch an, nachdem die Vegetationsstabilisierung überwunden ist; er sinkt dann aber mit der weiterhin abnehmenden Niederschlagsmenge wieder $a b$. Bei wieder zunehmendem Niederschlag müssen wir eine Umkehrung dieser Entwicklung erwarten, d. h. ein erneutes Aktivitätsmaximum vor der Stabilisierung der Oberfläche durch Vegetation. Die Aktivitätszeit ist dann dreigeteilt: 
Zwischen $\mathrm{zwei}$ Aktivitätsmaxima ist eine Trockenzeit eingeschaltet, die neben der geringeren Abspülungsintensität auch durch starke äolische Aktivität gekennzeichnet sein kann. Eine größere Zahl von Geländebefunden aus den Subtropen deutet darauf hin, daß die Aktivitätszeiten nicht symmetrisch, sondern in hohem Maße asymmetrisch aufgebaut waren, etwa wie in Abb. 2 dargestellt wurde. Die Trockenzeit ist gegen das Ende des Klimazyklus verschoben und das Aktivitätsmaximum II deutlich schwächer (weil kürzer) als das Aktivitätsmaximum I; im Extremfall kann das Aktivitätsmaximum II infolge sehr hohen Asymmetriegrades fast vollständig ausfallen ${ }^{6}$ ).

Was die letzte Kaltzeit im Mittelmeergebiet betrifft, so sprechen sich auch BuTzER (1958) und Messerli (1967) für ein sehr frühes Maximum der Abspülungswirkung aus. Ahnliche Verhältnisse liegen auch in Jordan:en vor, wo die detaillierten Untersuchungen von Huckriede \& Wiesemann (1968) zu einem Vergleich anregen. Nach diesen Autoren gehören die jüngsten großen Seehochstände Jordaniens ins Alt- und Mittelwürm (LisanSee, „Pluvial-See“ von El Jafr); danach folgte eine sehr ausgeprägte Trockenzeit: (PostLisan-Trockenzeit“) mit starker äolischer Aktivität (Windschliffe, Deflation, Dünen) und anschließend - noch im Jungwürm - wieder stärkere fluviale Aktivität mit Bildung der Regenpfanne von El Jafr, Einschneiden von Wadis in die „Pluvialkalke“ und Ablagerung von Schottern und Lehmen in anderen Wadisystemen, während in der Gegenwart innerhalb der Regenpfanne „nur noch geringfügig in den obersten Lagen bei Winterregen umgelagert wird“ (S. 82/83). Die offensichtliche Ahnlichkeit dieser Klimafolge, auch was die Asymmetrie der Klimawelle angeht, setzt allerdings ein Zusammenfallen von Seehochständen und starker Hangabtragung voraus (s. o.); Huckriede (1970) denkt bezüglich der Bildung des Lisan-Sees in Einklang mit unserer Ableitung an ausgeprägte winterliche Starkregen. Für das zweite Aktivitätsmaximum berichten auch HuCKRIEdE \& Wiesemann von verstärkter Waditätigkeit. Eine scheinbare Abweichung besteht darin, daß auch das Holozän als arid gilt und durch Dünenbildung charakterisiert wird, während nach Abb. 2 in der holozänen Stabilitätszeit relativ humide Verhältnisse herrschen sollten. Diese Abweichung liegt darin begründet, daß das Haupteinzugsgebiet des El Jafr-Beckens in niederschlagsreicheren Gebieten liegt und das Diagramm folglich vorwiegend für diese und weniger für das Becken selbst gilt.

In Mitteleuropa ist die Klimawelle der Kaltzeit-Warmzeit-Zyklen deutlich durch Sekundär- und Tertiäroszillationen überlagert, die zu einer Gliederung der Kaltzeiten in Stadiale und Interstadiale führt. Dasselbe Phänomen ist auch außerhalb der ehemaligen Periglazialgebiete festzustellen; eigene Untersuchungen an mächtigen Hang- und Schwemmfächersedimenten auf den Balearen und in Marokko ergaben nämlich eine ebenso reiche Untergliederung durch fossile Böden wie z.B. in unseren mitteleuropäischen Lößprofilen. Daraus muß geschlossen werden, daß die Sekundär(usw.)oszillationen sich wie die Primäroszillationen nicht nur thermisch, sondern auch auf die Niederschlagsverteilung auswirken und $\mathrm{zu}$ - wenn auch z. T. nur kurzfristigen - Stabilitätszuständen innerhalb der Kaltzeiten führten. Da in den Subtropen und Tropen weniger unterschiedliche Temperaturen, sondern weitaus stärker unterschiedliche Durchfeuchtung die typologische und intensitätsmäßige

6) Diese Asymmetrie hat ihre Parallele in Mitteleuropa, wo schon seit längerem bekannt ist, daß die größte Kälte und Trockenheit innerhalb der letzten Kaltzeit erst für das Jungwürm kennzeichnend ist (vgl. LožEK 1964). Nach KuKLA (1969), der einen gleichartigen klimatischen Aufbau aller Glazialzyklen annimmt, gilt das entsprechend auch für alle anderen Kaltzeiten. KUKLA (1969) begründet die Asymmetrie der Klimawelle damit, daß gegenüber dem auslösenden Prozeß der Klimaveränderung der Aufbau der Inlandeismassen beträchtlich nachhinkt und daß deswegen alle Folgeerscheinungen (stärkste Abkühlung aufgrund größerer Albedo, stärkste Trockenheit aufgrund geringster Verdunstung bei stärkster Abkühlung) ebenfalls gegen das Ende eines Glazialzyklus verschoben sind. Auch FLOHN (1970) leitet aus einem rein geophysikalischen Modell ab, daß wohl in jeder Kaltzeit das Hochglazial und vielleicht auch das Spätglazial durch eine ausgesprochen aride Phase von weltweiter Verbreitung charakterisiert sind. 
Ausbildung fossiler Böden bedingen, ist im Gegensatz zu Mitteleuropa oft nicht festzustellen, ob diese nun einer Warmzeit entstammen, oder ob sie aufgrund einer Sekundäroszillation innerhalb einer Kaltzeit gebildet wurden.

Ohne eine die Primäroszillationen untergliedernde Feinstratigraphie, wie sie in den Subtropen und Tropen erst in Ansätzen besteht, kann bei dieser Problemlage nicht sicher geklärt werden, ob die pleistozänen Klimaschwankungen in weiten Gebieten der Erde gleichartige Auswirkungen hatten oder nicht. Monod (1963) stellt dementsprechend einem zusammenfassenden Aufsatz über "The late Tertiary and Pleistocene in the Sahara " die Außerung WaYlands (1933) „I fear you are on rather ticklish ground with the Pluvials. We have yet much to learn... we need more facts " als Motto voran und schreibt im Text (S. 119): „The literature, however considerable, deals more with theories, opinions, and suppositions than with observed facts. These themselves are often subject to interpretations which are hazardous, ambivalent, or even impossible..." (vgl. auch Frenzel 1967, S. 183). In diesem Sinne sollte dieser Aufsatz weniger eine neue "Pluvial-Theorie“ zum Inhalt haben, sondern vielmehr ein Beitrag zur paläoklimatischen Deutung von „observed facts“ sein, da B es nämlich unumgänglich ist, Bodenbildung und morphodynamische Aktivität grundsätzlich voneinander zu trennen und nicht etwa unter demselben Begriff („Pluvial“) zu subsumieren.

Diese Unterscheidung könnte z. B. auch die verbreitete Vorstellung eines zeitlichen Alternierens von "Pluvialen“ in den Gebieten nördlich und südlich der Sahara (u.a. Balout 1952, Tricart 1956, Büdel 1963) hinfällig machen, denn nördlich der Sahara sind vorwiegend Hinweise für morphodynamische Aktivität und in den wechselfeuchten Tropen solche für Bodenbildung als „Pluvial“indikatoren verwandt worden. Aufgrund unserer Unterscheidung in Stabilitäts- und Aktivitätszeiten gelangt man jedoch eher zu der umgekehrten Vorstellung weitreichender Parallelität zwischen den Subtropen und Tropen, denn in beiden Gebieten herrschen heute Stabilitätsbedingungen mit Bodenbildung, während die letzte Kaltzeit in beiden Zonen (wenigstens zeitweise) durch morphodynamische Aktivität und eine ausgeprägte Trockenzeit ausgezeichnet war (FLOHN 1970). Inwieweit jedoch die Aktivitätsmaxima bzw. durch Sekundäroszillationen mit Bodenbildung getrennte Aktivitätsphasen weiträumig gleichaltrig sind, läßt sich zur Zeit nicht sicher abschätzen. Selbst wenn nämlich die Aktivitätszeiten im großen gleichzeitig sind, müssen die Aktivitätsmaxima unterschiedlicher Gebiete auch nach den aus Abb. 2 zu ziehenden Folgerungen nicht streng gleichzeitig sein. So könnte nämlich in niederschlagsreichen Gebieten mit großer Kampfkraft der Vegetation die Aktivitätszeit erst relativ spät einsetzen und ihr Maximum bei stärkster Niederschlagsverminderung erreichen, während in trockeneren Gebieten Teilaktivität und Aktivität schon frühzeitig herrschen, während der maximalen Niederschlagserniedrigung jedoch eine Trockenzeit zwei Aktivitätsmaxima voneinander trennt (Kurve N 2).

Die bisherigen Betrachtungen gelten zudem mehr oder weniger nur für die Gebiete, in denen die Oberfläche bei anthropo-zoogen ungestörter Vegetation gegenwärtig stabilisiert wäre ( $>100-150 \mathrm{~mm}$ Niederschlag). In den heute niederschlagsärmeren Gebieten (Halb- und Vollwüstenräume) müssen sowohl frühere Bodenbildungs- als auch Aktivitätszeiten niederschlagsreicher gewesen sein als heute (s. o.). Die gegenwärtige morphodynamische Stabilität auch der ariden Gebiete läßt darauf schließen, daß die Bildungszeiten der Paläoböden in weltweit sich auswirkenden Stabilitätszeiten mit wesentlich größerer Humidität als heute erfolgte ${ }^{7}$ ), in denen der Wüstenbereich der Sahara also sowohl von Norden als auch von Süden eingeengt war. Ein positiver Hinweis dafür ist z. B. darin zu

7) etwa in auf Sekundäroszillationen beruhenden Stabilitätszeiten mit temperaturbedingt geringer Verdunstung und dadurch höherer Humidität (vgl. Frenzel 1967)? 
sehen, daß das postglaziale Klimaoptimum sowohl in den Mittelbreiten (Frenzel 1967) als auch nördlich und südlich der Sahara (BUTZER 1958) feuchter war als heute. Umgekehrt erfolgte in den kaltzeitlichen Trockenphasen eine beiderseitige Ausdehnung der Sahara. Problematisch bleiben jedoch die Aktivitätszeiten der ariden Gebiete, die sich nur durch gleichzeitige Akzentuierung der Niederschlagsverteilung und Niederschlagserhöhung erklären lassen. Falls solche Bedingungen, die nicht in Abb. 2 aufgenommen worden sind, in größeren Gebieten herrschten, muß damit gerechnet werden, daß morphodynamische Aktivität zwar auch auf semiaride Gebiete übergriff, aber in stärker humiden Gebieten die Vegetationsstabilisierung nicht überwunden werden konnte. Darauf folgt ein Alternieren der Aktivitätsmaxima zwischen den Trockengebieten einerseits und den Feuchtgebieten andererseits ${ }^{8}$ ), was sowohl die Subtropen als auch die Tropen anbetrifft.

Der Mangel an „facts“, der im wesentlichen auf dem Fehlen einer Feinstratigraphie beruht, läßt keine Entscheidungen zu und verbietet zugleich die Fortführung dieser hypothetischen Erörterungen. Es erschien mir jedoch wesentlich, anzudeuten welche Konsequenzen aus einer Aufspaltung des mehrdeutigen Pluvialbegriffes gezogen werden müssen und in welcher Richtung zukünftige Forschungsergebnisse erwartet werden können.

Literaturverzeichnis

Alia Medina, M. u. Riba, O.: Livret - Guide de l'excursion $C_{4}$ : Manzanares et Tolède. INQUA V. Congr. Intern., Madrid-Barcelona 1957.

Balout, L.: Pluviaux interglaciaires et Préhistoire saharienne. - Trav. Inst. Rech. Sahar., 8, 9-21, Algier 1952.

Bartels, G.: Geomorphologische Höhenstufen der Sierra Nevada de Santa Marta (Kolumbien). Gießener Geogr. Schr., 21, Gießen 1970.

Beug, H. J.: Probleme der Vegetationsgeschichte in Südeuropa. - Ber. d. Dtsch. Botan. Ges., 80, 682-689, Berlin-Steglitz 1968.

Bigarella, J. J.: Slope development in south eastern and southern Brazil. - Z. f. Geomorph., 10, 150-160, Berlin 1966.

BRüCKNER, W.: The mantle rock ("laterite") of the Gold Coast and its origin. - Geol. Rdsch., 43, 307-327, Stuttgart 1955.

Brunnacker, K.: Affleurements de loess dans les régions Nord-Méditerrannéennes. - Revue de Géographie et de Géologie dynamique, 11, 325-334, Paris 1969.

BrunNaCker, K. \& Ložex, V.: Löß-Vorkommen in Südostspanien. - Z. f. Geomorph., 13, 297316, Berlin 1969.

Bonatri, E.: Late Quaternary pollen sequences from Central Italy. - Pollen et Spores, 4, 335336, Paris 1962.

BüDEL, J.: Die pleistozänen und quartären Pluvialzeiten der Sahara. - Eiszeitalter u. Gegenwart, 14, 161-187, OOhringen/Württ. 1963.

- : Pedimente, Rumpfflächen und Rückland-Steilhänge. - Z. f. Geomorph. N. F., 14, 1-57, Berlin-Stuttgart 1970.

Butzer, K.: Quaternary Stratigraphy and Climate in the Near East. - Bonner Geograph. Abh., 24, 157, Bonn 1958.

- : Paleoclimatic Implications of Pleistocene Stratigraphy in the Mediterranean Area. - Ann. New York Acad. Sci., 95, 449-456, New York 1961.

- : Pleistocene Geomorphology and Stratigraphy of the Costa Brava Region (Catalonia). Akad. Wiss. Lit. Mainz, Abh. Math.-Kat. K., Jg. 1964, Nr. 1, Mainz 1964 (1964a).

- : Climatic-geomorphologic interpretation of Pleistocene Sediments in the Eurafrican Subtropics. - African Ecology and Human Evolution (Hrsg.: Howell \& Bourlière), 1-27, London 1964 (1964b).

Butzer, K. \& Hansen, C. L.: Desert and River in Nubia. Madison 1968.

Coque, R.: La Tunisie présaharienne. Paris 1962.

8) Zwar nicht identische, aber in manchem ähnliche Vorstellungen entwickelte ANDREs (Kolloquiumsvortrag Geograph. Inst. Frankfurt, April 1970, über Südmarokko). 
FLOHN, H.: Zur meterologischen Interpretation der pleistozänen Klimaschwankungen. - Eiszeitalter u. Gegenwart, 14, 153-160, Ơhringen/Württ. 1963.

— : frdl. briefl. Mittlg. Februar 1970.

FöLSTER, H.: Morphogenese der südsudanesischen Pediplane. - Z. f. Geomorph. N. F., 8, 393-423, Berlin 1964.

- : Slope Development in SW-Nigeria during Late Pleistocene and Holocene. - Gießener Geogr. Schr., 20, 3-56, Gießen 1969.

FrenzeL, B.: Die Klimaschwankungen des Eiszeitalters. Braunschweig 1967.

HAGEdorn, H.: Beobachtungen an Inselbergen im westlichen Tibesti-Vorland. - Berliner Geogr. Abh., 5, 17-22, Berlin 1967.

Hammen, Th. van der \& Wijmstra, T. A. \& Molen, W. H. van der: Palynological study of a very thick peat section in Greece, and the Würmglacial vegetation in the Mediterranean region. - Geologie en Mijnbouw, 44, 37-39, S’Gravenhage 1965.

Haude, W.: Erfordern die Hochstände des Toten Meeres die Annahme von Pluvial-Zeiten während des Pleistozäns? - Meteorol. Rdsch., 22, 29-40, Berlin 1969.

Herrmann, R.: Methoden und Ergebnisse einer hydrographischen Landesaufnahme von Ostnigeria. - Erdkunde, 22, 89-100, Bonn 1968.

- : Zur regionalhydrologischen Analyse und Gliederung der nordwestlichen Sierra Nevada de Santa Marta (Kolumbien). Habilitationsschr. Math.-Nat. Fak. Univ. Gießen, im Druck in Gießener Geogr. Schr.

Hövermann, J.: Hangformen und Hangentwicklung zwischen Syrte und Tschad. L'Evolution des Versants; Les Congrès et Colloques de l'Université de Liège, 40, 139-156, Liège 1967.

Huckriede, R.: frdl. briefl. Mittlg. Februar 1970.

Huckriede, R. \& Wiesemann, G.: Der jungpleistozäne Pluvial-See von El Jafr und weitere Daten zum Quartär Jordaniens. - Geologica et Palaeontologica, 2, 73-95, Marburg 1968.

Kukla, J.: Die zyklische Entwicklung und die absolute Datierung der Löß-Serien. - Periglazialzone, Löß und Paläolithikum der Tschechoslowakei, 75-95, Brno 1969.

LANGBein, W. B. \& Schumm, S. A.: Yield of sediment in relation to mean annual precipitation. Amer. Geophys. Union Trans., 39, 1076-1084, Washington 1958.

Lauer, W.: Humide und aride Jahreszeiten in Afrika und Südamerika und ihre Beziehungen zu den Vegetationsgürteln. - Bonner geogr. Abh., 9, 15-66, Bonn 1952.

Leopold, L. B.: Rainfall frequency, an aspect of climatic variation. - Trans. Amer. Geophys. Union, 32, 347-357, Washington 1951.

Leopold, L. B., Leopold, E. B. \& Wendorf, F.: Some climatic Indicators in the Period A. D. 1200-1400 in New Mexico. Changes of Climate (Arid Zone Research, UNESCO), 265-280, Paris 1963.

LožEK, V.: Quartärmollusken der Tschechoslowakei. - Rospravy Ústředního Ústavu Geologického, 31, Praha 1964.

Mabesoone, J. M.: Relief of northeastern Brazil and its correlated sediments. - Z. f. Geomorph., 10, 419-453, Berlin 1966.

MenÉndez Amor, J. \& Florschütz, F.: Results of the preliminary palynological investigation of samples from a $50 \mathrm{~m}$ boring in Southern Spain. - Bol. R. Soc. Española Hist. Nat. (Geol.), 62, 251-255, Madrid 1964.

Mensching, H.: Karst und Terra rossa auf Mallorca. - Erdkunde, 9, 188-196, Bonn 1955.

—: Die regionale und klimatisch-morphologische Differenzierung von Bergfußflächen auf der Iberischen Halbinsel (Ebrobecken - Nordmeseta - Küstenraum Iberiens). - Würzburger Geogr. Arb., 12, 141-158, Würzburg 1964 (1964a).

- : Zur Geomorphologie Südtunesiens. - Z. f. Geomorph., 8, 424-439, Berlin 1964 (1964b).

Messerli, B.: Die eiszeitliche und die gegenwärtige Vergletscherung im Mittelmeerraum. - Geographica Helvetica, 1967, 105-228, Bern 1967.

Meyer, B. \& Moshrefi, N.: Experimente zur Entstehung von Einwaschungs-Tonlamellen in Böden und Sedimenten durch Fließvorgänge im ungesättigten Feuchtezustand. - Masch.-Schr. Mskr. 1969, im Druck in Göttinger Bodenkdl. Ber.

Monod, Th.: The late Tertiary and Pleistocene in the Sahara. - African Ecology and Human Evolution (ed. F. C. Howell \& F. Bourlière), 117-229, London 1964.

NyE, P.: Some soil-forming processes in the humid tropics: I A field study of a catena in the West African forest. - J. Soil Sci., 5, 7-21, Oxford, Univ. Press 1954.

- : IV The action of the soil fauna. - J. Soil Sci., 6, 73-83, Oxford 1955.

De Ploey, J.: Position géomorphologique, genèse et chronologie de certains dépôts superficiels au Congo occidental. - Quaternaria VII, 131-154, Roma 1965. 
RiBA, O.: Livret-guide de l'excursion $\mathrm{L}_{2}$ : Terrasses du Manzanares et du Jarama aux environs de Madrid. — INQUA V. Congr. Intern. Madrid-Barcelona 1957.

Rognon, P.: Climatic Influences on the African Hoggar During the Quaternary, Based on Geomorphologic Observations. - Ann. Assoc. Amer. Geogr., 57, 115-127, Washington 1967.

RohDENBurg, H.: Hangpedimentation und Klimawechsel als wichtigste Faktoren der Flächenund Stufenbildung in den wechselfeuchten Tropen an Beispielen aus Westafrika, besonders aus dem Schichtstufenland Südost-Nigerias. - Gießener Geogr. Schr., 20, 57-152, Gießen 1969.

- : Hangpedimentation und Klimawechsel als wichtigste Faktoren der Flächen- und Stufenbildung in den wechselfeuchten Tropen. - Z. f. Geomorph. N. F., 14, 58-78, Berlin-Stuttgart 1970.

Rohdenburg, H. \& Sabelberg, U.: „Kalkkrusten“ und ihr klimatischer Aussagewert - neue Beobachtungen aus Spanien und Nordafrika. - Göttinger Bodenkdl. Ber., 7, 3-26, Göttingen 1969 (1969a).

- : Zur landschaftsökologisch-bodengeographischen und klimagenetisch-geomorphologischen Stellung des westlichen Mediterrangebietes. - Göttinger Bodenkdl. Ber., 7, 27-47, Göttingen 1969 (1969b).

Schumm, S. A.: Quaternary Palaohydrology. The Quaternary of the United States (Hrsg. H. E. Wright \& D. G. Frey), 783-794, Princeton 1965.

Tricart, J.: Tentative de corrélation des périodes pluviales africaines et des périodes glacaires. C. R. Somm. Soc. Géol. France, 9 u. 10, 164-167, Paris 1956.

- : Le modelé de régions chaudes forêts et savanes. - Traité de Géomorph., Tome, 5, Paris 1965.

Vincent, P. L.: Les formations meubles superficielles au Sud du Congo et au Gabon. vgl. Vogt \& Vincent (1966).

Virgili, C. \& Zamarreno, J.: Le Quaternaire continental de la plaine de Barcelone. - INQUA V. Congr. Intern. Livretguide de l'excursion $B_{1}$ : Environs de Barcelone et Montserrat, 7-16, Madrid-Barcelone 1957.

Vogt, J.: Le complex de la stone-line. Mise au point. vgl. Vogt \& Vincent (1966).

Vogt, J. \& Vincent, P. L.: Terrains d'altération et de recouvrement en zone intertropicale. Bull. du B. R. G. M., 4, 1-111, Paris 1966.

WIChE, K.: Geomorphologische Studien in Südostspanien (Provinz Murcia). - Mitt. Ơsterr. Geogr. Ges., 101, 390-395, Wien 1959.

- : Beiträge zur Formenentwicklung der Sierren am unteren Segura (Südostspanien). - Mitt. d. Österr. Geograph. Ges., 103, 125-157, Wien 1961.

Manuskr. eingeg. 22. 5. 1970.

Anschrift des Verf.: Wiss.R. u. Prof. Dr. H. Rohdenburg, Geographisches Institut der Justus-LiebigUniversität, 63 Gießen, Landgraf-Philipp-Platz 2, Neues Schloß. 